Interleukin-22 levels are increased in gastrointestinal graft-versus-host disease in children

Interleukin-22 (IL-22) is a member of the IL-10 cytokine family, is induced by many different environmental and endogenous signals, ${ }^{1}$ and is produced by adaptive and innate immune cells, including innate lymphoid cells (ILCs). ${ }^{2}$ IL-22 binds to receptors on the epithelial cells of the intestinal border, making IL-22 an important mediator between immune and epithelial systems. ${ }^{3}$ IL-22 has both protective and inflammatory properties, depending on context, and the role of IL-22 in GvHD is not fully understood, and may be dependent on multiple factors. ${ }^{4}$

In the gastrointestinal tract, IL-22 works at the epithelial border to promote tissue repair and healing through three mechanisms: induction of MUC proteins and increased mucous production in the epithelial border, increased cell proliferation and survival, and increased expression of antimicrobial proteins in the intestinal border, such as reg3 $\alpha^{3,5}$ Previous studies have shown that IL-22 is critical for the wound healing response, as deficiency has been associated with higher susceptibility to dextran sulfate sodium (DSS)-induced colitis in mice. ${ }^{6}$ However, IL-22 also has pro-inflammatory properties with increased levels leading to inflammation and hyperproliferation of cells resulting in negative effects on tissues. ${ }^{7}$

In murine transplant models, IL-22 produced from recipient-derived ILCs leads to improved epithelial recovery and IL-22 deficient transplant recipients have increased GvHD mortality and greater loss of intestinal stem cells (ISCs) during GvHD. ${ }^{8}$ IL-22 administered to mice after allogeneic HSCT led to a decrease in GvHD pathology in the small intestine, large intestine, and liver as well as decreased intestinal crypt apoptosis in both small and large intestine. ${ }^{9}$ Moreover, ISC recovery and donor marrow-derived T-cell development were improved in mice that received IL-22 following HSCT compared to those who did not receive IL- $22 .{ }^{9}$ In contrast, IL-22 produced by donor-derived $\mathrm{CD} 4^{+} \mathrm{T}$ cells led to worsened GI pathology and increase in GI GvHD incidence. $^{8}$

Our study aimed at determining the role of circulating IL-22 levels in GvHD in children undergoing hematopoietic stem cell transplant since most of the previous work has been performed in mouse transplant models. We hypothesized that IL-22 levels would be elevated in patients with GI GvHD, and the IL-22 would be of recipient origin, promoting healing and reducing mortality. We tested this hypothesis in a cohort study of children receiving allogeneic HSCT and show that IL-22 levels are elevated in GI GvHD and elevated levels are likely of recipient origin. Children were enrolled in a prospective cohort study, the Cincinnati Children's Hospital Medical Center BMT repository. Prospective weekly blood, stool, and urine samples are collected for biological studies of complications of transplantation from all consented cases. The institutional review board approved the study. One hundred and fourteen patients were included in the current analysis (Table 1). Clinical data were abstracted from the institutional transplant database and from the medical record. The treating physician diagnosed acute GI GvHD and supported this diagnosis with tissue biopsies whenever applicable and graded it according to the modified Glucksberg criteria. ${ }^{10}$

IL-22, IL-22 binding protein (IL-22BP), Reg3 $\alpha$, and IL-17 levels were measured in patient plasma at day 30 post-transplant using ELISA kits (R\&D Systems and Cloud Clone) as directed by manufacturer instructions.

To assess the cellular source of IL-22 production, cryopreserved peripheral blood mononuclear cells from day +30 post-transplant were thawed and incubated with Phorbol 12 Myristate 13 Acetate (PMA); SIGMA, Saint Louis, USA), ionomycin (SIGMA, Saint Louis, USA) and Brefeldin A (SIGMA, Saint Louis, USA) for $6 \mathrm{~h}$ at $37^{\circ} \mathrm{C}$ and $5 \% \mathrm{CO} 2$. Cells were stained against CD3 (Biolegend, San Diego, CA) and CD8 (BD Biosciences, San Jose CA), washed followed by permeabilization and fixation using

Table 1. Patient and Transplant Demographics ( $n=114)$.

$\begin{array}{lc}\text { Characteristic } & \text { Value } \\ \text { Age } & \\ \text { Median (year) } & 8 \\ \text { Range (year) } & 0.4-32 \\ \text { Diagnosis } & \\ \text { Malignancy } & 32(28 \%) \\ \text { Immune Deficiency } & 39(34 \%) \\ \text { Bone Marrow Failure } & 43(38 \%) \\ \text { Conditioning regimens } & \\ \text { Myeloablative } & 67(59 \%) \\ \text { Reduced Intensity } & 47(41 \%) \\ \text { Stem Cell Source } & \\ \text { BM* } & 95(84 \%) \\ \text { PBSC } & 14(12 \%) \\ \text { CB } & 5(4 \%)\end{array}$

\section{Cell Dose}

Median $6.8 \times 10^{8}$ cells $/ \mathrm{kg}$

Range $\quad 15.4 \times 10^{6}$ cells $/ \mathrm{kg}-32.75 \times 10^{8} \mathrm{cells} / \mathrm{kg}$

\begin{tabular}{ll} 
Donor & \\
Related & $42(37 \%)$ \\
Unrelated & $72(63 \%)$ \\
\hline
\end{tabular}

Match

BM and PBSC

$\begin{array}{cc}10 / 10 & 85(75 \%) \\ 9 / 10 & 21(18 \%) \\ 8 / 10 & 3(2 \%) \\ \text { UCB } & \\ 6 / 6 & 2(2 \%) \\ 5 / 6 & 1(1 \%) \\ 4 / 6 & 2(2 \%)\end{array}$

GvHD Prophylaxis

\begin{tabular}{lc} 
CSA + Other & $101(89 \%)$ \\
Tacro + Other & $3(3 \%)$ \\
Ex-vivo T-cell depletion & $9(7 \%)$ \\
Other & $1(1 \%)$ \\
\hline GvHD any site & $50(44 \%)$ \\
Grade 1 & $9(18 \%)$ \\
Grades 2-4 & $41(82 \%)$ \\
GI GvHD & $31(27 \%)$ \\
Grade 1 & $3(10 \%)$ \\
Grades 2-4 & $28(90 \%)$
\end{tabular}

*BM: Bone Marrow; PBSC: peripheral blood stem cell;UCB: umbilical cord blood; CSA: Cyclosporine; Tacro: Tacrolimus. 
A

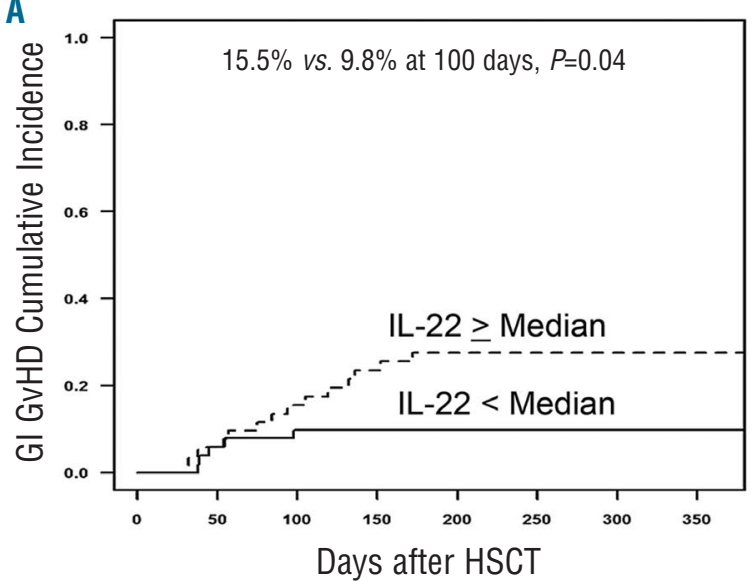

B

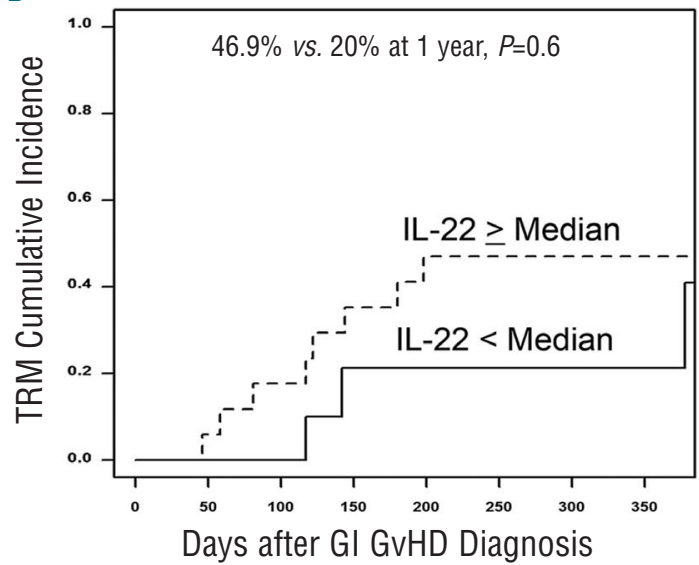

Figure 1. IL-22 levels and transplant outcomes. (A) Cumulative incidence of new onset GI GvHD within 100 days of transplant was increased in patients with IL-22 levels above the median compared to those below the median (15.5\% vs. 9,8\% at 100 days, $P=0.04$ ). (B) TRM amongst patients with Gl GvHD was not statistically different between patients with IL-22 levels above the median compared to those with IL-22 levels below the median at 1 year post-transplant ( $46.9 \%$ vs. $20 \%$ at 1 year, $P=0.6$ ).

the commercially available Cytofix/Cytoperm kit (BD Biosciences San Jose, CA, USA) according to the manufacturer's protocol and then stained against IL-22 (Biolegend, San Diego CA, USA). Sample acquisition was done on a FACS Canto II flow cytometer (BD Biosciences, San Jose, CA) and analyzed using FSC express version 5.0 (De Novo Software, Glendale, CA, USA).

Continuous data are summarized as median (range). Overall Survival and cumulative incidence were estimated using Kaplan-Meier; differences in above and below the median were assessed using the log-rank test and Gray's method of competing risks, where death and relapse were treated as competing events, respectively. Correlation was reported and tested as Spearman's rankorder correlation. Significance was assessed at the 0.05 level. All computations were done in $\mathrm{R}$ (version 3.2.4, Vienna, Austria).

The cumulative incidence of new onset acute GI GvHD after day +30 was higher in patients with an IL-22 level above the median at day +30 compared to patients with an IL-22 level below the median at day $+30(15.5 \%$ vs.
A

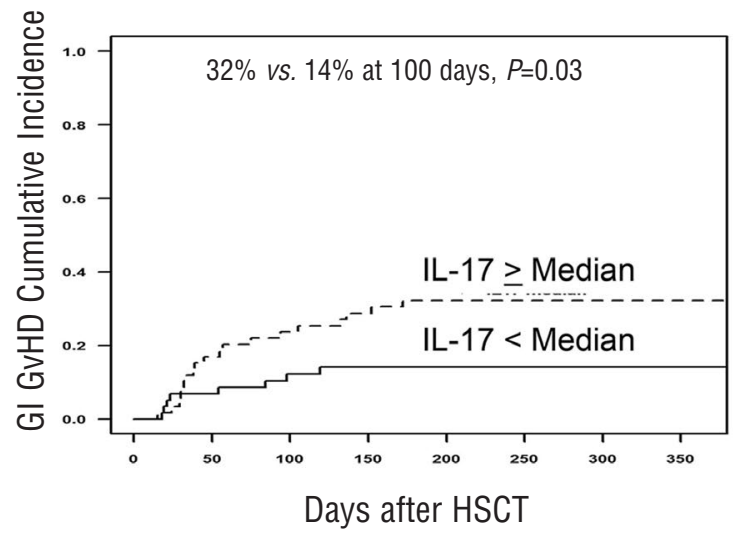

B

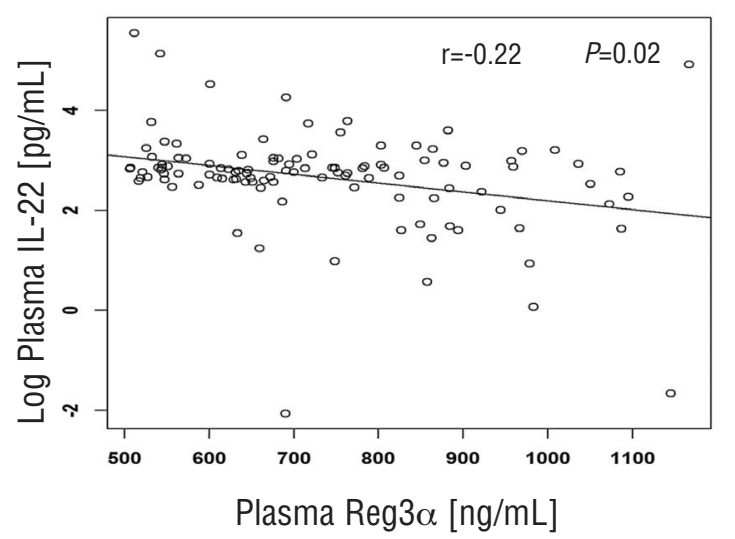

Figure 2. IL-17 and Reg3 $\alpha$ levels and transplant outcomes. (A) The cumulative incidence of GI GvHD 100 days after transplant was increased in patients with an IL-17 level above the median compared to those with an IL-17 level below the median ( $32 \%$ vs. $14 \%, P=0.03$ ). (B) Reg3 $\alpha$ levels were negatively correlated with IL-22 levels at day 30 post-transplant $(r=-0.22, P=0.02)$.

$9.8 \%, P=0.04$; Figure $1 \mathrm{~A})$. TRM amongst patients that developed acute GI GvHD IL-22 levels above the median was $46.9 \%$ compared to patients with IL-22 levels below the median of $20 \%$ at 1 year, $P=0.6$, Figure $1 \mathrm{~B}$.

IL-22 binding protein (IL-22BP) is a soluble, potent inhibitor of IL-22 and is thought to regulate its downstream effects and has been found to be enhanced during active colitis of patients with IBD. ${ }^{11,12}$ We hypothesized that IL-22BP levels would be elevated in patients with GI GvHD and that these levels would be inversely correlated with IL-22 levels. IL-22BP levels at day 30 post-transplant had a negative correlation with IL-22 levels at day 30 post-transplant $(\mathrm{r}=-0.2, P=0.03)$ but had no association with the development of GI GvHD.

Exacerbated or uncontrolled activity of IL-22 can cause inflammation, specifically when IL-22 functions in concert with interleukin-17 (IL-17), a pro-inflammatory cytokine produced by Th17 cells. ${ }^{13}$ We hypothesized that IL-17 levels would be higher in patients with GI GvHD and positively correlated with IL-22 levels. In agreement with our hypothesis, IL-17 levels above the median at day +30 post-transplant were associated with an 
increased incidence of GI GvHD compared to patients with IL-17 levels below the median (32\% vs. $14 \%$ at 100 days, $P=0.03$, Figure $2 \mathrm{~A})$. IL-17 levels were also correlated with IL-22 levels at day 30 post-transplant $(r=0.43$, $P<0.0001$ )

Reg3 $\alpha$ is an anti-microbial protein whose expression in the intestinal border is modified by IL-22. We hypothesized that children with higher IL-22 levels would have higher levels of Reg $3 \alpha$. In contrast to our hypothesis, we found a negative correlation between IL-22 and reg $3 \alpha$ $(\mathrm{r}=-0.22, P=0.02$, Figure $2 \mathrm{~B})$. We also found a negative correlation between $\operatorname{Reg} 3 \alpha$ and IL-17 at day 30 posttransplant $(\mathrm{r}=-0.47, P<0.0001)$.

We selected six patients with high levels of IL-22 at day +30 after HSCT and analyzed frozen peripheral blood mononuclear cells at a similar time point. Patients with at least $250 \mathrm{CD}^{+}$and $\mathrm{CD}^{+} \mathrm{T}$-cell events were eligible for analysis $(n=5)$. Four of the five patients had donor chimerism $>99 \%$ by day +30 post-transplant, and 1 patient had a donor chimerism of $93 \%$ at day +30 posttransplant. No patient had detectable IL-22 expression before or after incubation with PMA, ionomycin and brefeldin A in CD8 or $\mathrm{CD}^{+} \mathrm{T}$ cells suggesting that IL22 was not being produced by donor $T$ cells.

We report that the incidence of GI GvHD was higher in patients with IL-22 levels above the median compared to patients without GI GvHD. In our study, Reg3 $\alpha$ levels were negatively correlated with IL-22 levels post-transplant, suggesting a reciprocal relationship between the two molecules. Notably, reg3 $\gamma^{-1-}$ mice have higher levels of IL-22 in the intestine compared to wild type mice. This finding is thought to reflect increased microbial translocation and inflammation in mice lacking reg $3 \gamma$, leading to stimulation of IL-22 production for healing. ${ }^{14}$ Our finding of a negative correlation between IL-22 and Reg3 $\alpha$ in the current study supports this hypothesis.

In our study, we saw no evidence of production of IL22 in donor-derived lymphocytes, supporting production from recipient cells. We speculate that the IL-22 we are measuring is produced by ILC3 cells in the intestine.

The preponderance of evidence from our study supports an overall beneficial role for elevated IL22 levels in GI GvHD. We propose a potential mechanism for elevated IL-22 levels in the post-transplant setting. Prior to transplant, the mucosal barrier is intact, covered with a layer of mucous containing antimicrobial peptides, such as Reg3 $\alpha$. ILC3s are present in the intestine, producing a basal level of IL-22 for intestinal homeostasis. The transplant preparative regimen injures the intestinal epithelial cells, decreasing Reg $3 \alpha$ levels, and increasing intestinal permeability to infectious and inflammatory molecules. During this inflammatory state, some patients develop GI $\mathrm{GvHD}$, which further injures the intestine leading to a decrease in ILC3 cell population. Decreased Reg3 $\alpha$ production leads to increased microbial translocation and inflammation, as found in previous murine studies, ${ }^{14}$ stimulating an increase in IL-22 production, promoting intestinal healing and repair.

In summary, we show that IL-22 levels are elevated in patients with GI GvHD without impacting mortality amongst patients with GI GvHD. Our results support the hypothesis that IL-22 is elevated in response to intestinal injury and is promoting intestinal healing. We recognize, however, that the role of IL-22 and other related molecules is quite complex in the post-transplant setting, and further studies are needed to fully elucidate mechanisms of action, which may vary importantly by context.

Dana T. Lounder, ${ }^{1}$ Pooja Khandelwal, ${ }^{1}$ Nicholas J. Gloude,' Christopher E. Dandoy, ${ }^{1}$ Sonata Jodele, ${ }^{1}$ Mario Medvedovic, ${ }^{2}$ Lee A. Denson, ${ }^{3}$ Adam Lane, ${ }^{1}$ Kelly Lake, ${ }^{1}$ Bridget Litts, ${ }^{1}$ Alyss Wilkey' and Stella M. Davies

${ }^{1}$ Division of Bone Marrow Transplant and Immune Deficiency, Cincinnati Children's Hospital Medical Center; ${ }^{2}$ Division of Biostatistics and Bioinformatics, Department of Environmental Health, University of Cincinnati and ${ }^{3}$ Division of Gastroenterology, Hepatology, and Nutrition, Cincinnati Children's Hospital Medical Center, OH, USA

Correspondence:dana.lounder@weirtonmedical.com doi:10.3324/haematol.2017.174771

Information on authorship, contributions, and financial \& other disclosures was provided by the authors and is available with the online version of this article at wWW. haematologica.org.

\section{References}

1. Xie $\mathbf{M H}$, Aggarwal S, Ho WH, et al. Interleukin (IL)-22, a novel human cytokine that signals through the interferon receptor-related proteins CRF2-4 and IL-22R. J Biol Chem. 2000;275(40):3133531339.

2. Takatori H, Kanno Y, Watford WT, et al. Lymphoid tissue inducerlike cells are an innate source of IL-17 and IL-22. J Exp Med. 2009;206(1):35-41

3. Sabat R, Ouyang W, Wolk K. Therapeutic opportunities of the IL-22IL-22R1 system. Nat Rev Drug Discov. 2014;13(1):21-38.

4. Lamarthee B, Malard F, Saas P, et al. Interleukin-22 in graft-versushost disease after allogeneic stem cell Transplantation. Front Immunol. 2016;7:148

5. Zindl CL, Lai JF, Lee YK, et al. IL-22-producing neutrophils contribute to antimicrobial defense and restitution of colonic epithelial integrity during colitis. Proc Natl Acad Sci USA. 2013;110(31):12768-12773.

6. Sugimoto K, Ogawa A, Mizoguchi E, et al. IL-22 ameliorates intestinal inflammation in a mouse model of ulcerative colitis. J Clin Invest. 2008;118(2):534-544

7. Andoh $\mathrm{A}$, Zhang Z, Inatomi $\mathrm{O}$, et al. Interleukin-22, a member of the IL-10 subfamily, induces inflammatory responses in colonic subepithelial myofibroblasts. Gastroenterology. 2005;129(3):969-984.

8. Hanash AM, Dudakov JA, Hua G, et al. Interleukin-22 protects intestinal stem cells from immune-mediated tissue damage and regulates sensitivity to graft versus host disease. Immunity. 2012;37(2):339350 .

9. Dudakov JA, Velardi E, Hua G, et al. IL-22 administration decreases intestinal GvHD pathology, increases intestinal stem cell recovery, and enhances immune reconstitution following allogeneic hematopoietic transplantation. Blood. 2013;122(21):290.

10. Glucksberg H, Storb R, Fefer A, et al. Clinical manifestations of graftversus-host disease in human recipients of marrow from HL-Amatched sibling donors. Transplantation. 1974; (4):295-304.

11. Pelczar P, Witkowski M, Perez LG, et al. A pathogenic role for T cellderived IL-22BP in inflammatory bowel disease. Science. 2016; 354(6310):358-362.

12. Martin JC, Beriou G, Heslan M, et al. IL-22BP is produced by eosinophils in human gut and blocks IL-22 protective actions during colitis. Mucosal Immunol. 2016;(2):539-549.

13. Eyerich S, Eyerich K, Cavani A, et al. IL-17 and IL-22: siblings, not twins. Trends Immunol. 2010;(9):354-361.

14. Loonen LM, Stolte EH, Jaklofsky MT, et al. REG3gamma-deficient mice have altered mucus distribution and increased mucosal inflammatory responses to the microbiota and enteric pathogens in the ileum. Mucosal Immunol. 2014;(4):939-947. 